\title{
Laparoscopic pyeloplasty: the updated McMaster University experience
}

\author{
Jihao Dong; ${ }^{*}$ Jaime Wong, MD; ${ }^{\xi}$ Ahmad Al-Enezi, MD;" Anil Kapoor, MD; ${ }^{\dagger} \mathrm{J}$. Paul Whelan, MD; ${ }^{\dagger}$ \\ Kevin Piercey, $M D_{;}^{;}$Edward D. Matsumoto, $M D^{\ddagger}$
}

See related article on page 393

\begin{abstract}
Objective: The open Anderson-Hynes procedure has an overall success rate of $90 \%$ for ureteropelvic junction obstruction. Laparoscopic pyeloplasty (LP) was developed to reduce morbidity and hospital stay while preserving the excellent results. We report on the results of our experience with laparoscopic pyeloplasty.

Methods: Between January 2001 and May 2006, 77 consecutive patients underwent LP performed by one of 4 surgeons at our institution. Patients were reassessed with ultrasound (U/S) or intravenous pyelogram (IVP) at 6 weeks. Diuretic renal scan and U/S or IVP were performed at 6 months, and subsequent follow-up included a U/S or IVP as well as clinical assessment. Patients were assessed for pain and hydronephrosis on radiologic imaging, clearance on diuretic renal scan $\left(\mathrm{T}^{1 / 2}\right)$ and differential renal function.
\end{abstract}

Results: We evaluated 73 patients. The mean patient age was 38 years (range 16-71 yr), the mean operating time was 218 minutes (range 110-409 min), and the mean blood loss was $57 \mathrm{~mL}$ (range $25-250 \mathrm{~mL}$ ). Mean hospital stay was 3.0 days (range $2-7$ d). The success rate was $90.4 \%$, and failures were mainly due to poor function after surgery ( 3 patients). Pyelolithotomy was performed concomitantly on 6 patients, which on average extended operative time by 36 minutes.

Conclusion: Our success rates are consistent with the LP experience of other centres and are comparable with rates for the open technique. Patients had short hospital stays, and complications were negligible. With experienced surgeons, LP should be the first-line treatment for ureteropelvic junction obstruction.

CUAJ 2008;2(4):388-92

\section{Introduction}

Ureteropelvic junction obstruction (UPJO) is a common urologic problem that has had excellent surgical treatment outcomes since Anderson-Hynes first described the dismembered pyeloplasty technique more than 50 years ago. The open Anderson-Hynes procedure continues to be the gold standard, with an overall success rate of $90 \%$. $^{1}$ To decrease the morbidity and hospital care costs associated with the large flank incision needed for the open procedure, a flurry of activity has been directed to developing minimally invasive surgical techniques to reduce morbidity while preserving excellent success rates. Endopyelotomy successfully reduced morbidity, but long-term follow-up revealed a lower success rate in comparison with the open procedure. ${ }^{2}$ In 1993, Schuessler ${ }^{3}$ first introduced laparoscopic pyeloplasty (LP), which has been shown to reduce hospital stay while offering success rates equivalent to those of the open procedure. Since our initial report 3 years ago, other centres have published their experiences with LP.4-7 Additionally, several new innovations have been described..$^{8-11}$ This paper summarizes our experience with LP, ${ }^{12}$ and briefly discusses the current status of LP.

\section{Methods}

After obtaining local research ethics board approval, we reviewed the charts of 77 consecutive patients who underwent LP at our institution between January 2001 and May 2006. All patients had UPJO confirmed with imaging and/or diuretic renal scan.

Laparoscopic transperitoneal dismembered Anderson-Hynes pyeloplasty is our procedure of choice. The surgery is performed through ports measuring $2 \times 10 \mathrm{~mm}$ and $2 \times 5 \mathrm{~mm}$. First, the renal pelvis is transected, then the ureteropelvic junction is resected, and then the proximal ureter is spatulated laterally. The anastomosis is completed with 2 different-coloured 4.0 vicryl sutures, with antegrade stent insertion done laparoscopically after the completion of the posterior wall anastomosis and before the completion of the anterior anastomosis. First, a 5-French angiocatheter is passed through a port in the abdomen and into the ureter, then a 0.035-inch guidewire is passed through the angiocatheter and into the bladder, and last, a 6-French double pigtail stent is advanced over the guidewire until it is in position and confirmed under fluoroscopy..$^{13}$ One of the surgeons removes the stent 2 weeks after surgery, and the other 3 surgeons remove the stent between 4 and 6 weeks after surgery.

Pyelolithotomy was performed if there were associated stones. After the renal pelvis was transected, stones were removed individually under direct laparoscopic vision with the use of standard laparoscopic graspers. 
Calyceal stones were removed with the introduction of a flexible cystoscope through one of the $10-\mathrm{mm}$ ports and the use of a standard stone basket. Resection of the ureteropelvic junction and reconstruction of the renal pelvis proceeded as normal.

At 6 weeks, patients were reassessed with ultrasound (U/S) or intravenous pyelogram (IVP). Diuretic renal scan and U/S or IVP were performed at 6 months, and subsequent follow-up included U/S or IVP as well as clinical assessment.

The LP was considered successful when patients had a complete resolution or marked reduction of pain and improvement of hydronephrosis on radiologic imaging or increased drainage on diuretic renal scan as measured by a reduction in diuretic renal scan clearance time $\left(\mathrm{T}^{1 / 2}\right)$ by a factor of 2 or by a $T^{1 / 2}$ value of less than 10 minutes postoperatively or postoperative increase in differential renal function (DRF) of greater than $10 \%$.

\begin{tabular}{lc}
\hline $\begin{array}{l}\text { Table 1. Characteristics of patients } \\
\text { with uteropelvic junction obstruction }\end{array}$ & $\begin{array}{c}\text { No. of } \\
\text { patients* }\end{array}$ \\
\hline & \\
Characteristic & 41 \\
\hline Sex & 36 \\
$\quad$ Male & $38(16-71)$ \\
Female & \\
Mean (and range) age, yr & 70 \\
Indication for surgery & 2 \\
$\quad$ Pain & 1 \\
\hline Obstruction & \\
\hline Decline in renal function & \\
\hline *Unless otherwise indicated. &
\end{tabular}

\section{Results}

Patient characteristics are presented in Table 1. Of the 77 patients, 73 were available for evaluation, representing the experience of 4 urologists at this institution. We excluded 4 patients because 3 were being followed by an out-of-province urologist and $1 \mathrm{LP}$ had been converted to an open procedure owing to difficulties with the anastomosis.

Of the 73 patients evaluated, 70 presented with flank pain, 2 were incidentally found to have obstruction during investigation for other diseases, and 1 was diagnosed by imaging during a workup for declining renal function. Most of the patients were treated for primary UPJO, with only 3 patients undergoing secondary repair after failure of previous endopyelotomy procedures.

The operative results are presented in Table 2. There was no difference in outcome between stent removal at 2 weeks or at 4-6 weeks. Mean followup was 13 (range 1-31) months. The choice of U/S or IVP depended on surgeon preference; 4 patients underwent both tests before and after surgery, and all patients received at least one imaging modality. Preoperative findings of obstruction included moderate-to-severe hydronephrosis on imaging, increased $\mathrm{T}^{1 / 2}$ and decreased DRF. U/S showed improvement of hydronephrosis in $43 / 50$ patients $(86.0 \%)$, and IVP showed improvement in $16 / 27$ patients (59.3\%). $T^{1 / 2}$ improved in 52/64 $(81.3 \%)$ patients. Most patients had DRF that was close to equal bilaterally on preoperative renal scan, but of the 4 patients who had significantly decreased function (less than $30 \%$ in the obstructed kidney) preoperatively, only 1 had a functional

Table 2. Operative results

\begin{tabular}{lc}
\hline Result & $\begin{array}{c}\text { No. (and \%) of } \\
\text { patients* }\end{array}$ \\
\hline Mean (and range) operating time, min & $218(110-409)$ \\
Mean (and range) blood loss, mL & $57(25-250)$ \\
Mean (and range) hospital stay, d & $3(2-7)$ \\
Mean (and range) follow-up, mo & $13(1-31)$ \\
Rate of crossing vessels, \% & 62.0 \\
Complete resolution or improvement of pain & $67 / 70(95.7)$ \\
Improvement in postoperative ultrasound & $43 / 50(86.0)$ \\
Improvement in postoperative intravenous pyelogram & $16 / 27(59.3)$ \\
Improvement in renal scan drainage & $52 / 64(81.3)$ \\
Overall success (improvement in pain and on imaging) & $66 / 73(90.4)$ \\
\hline *Unless otherwise indicated. & \\
\hline
\end{tabular}


Dong et al.

increase after surgery. The 3 patients who did not recover function all had preoperative DRF of less than $25 \%$.

Table 3 shows a summary of patient outcomes. Of the patients, 62 reported a complete resolution of pain; 5 additional patients reported improvement in pain with only mild discomfort that did not require further treatment. The overall success rate was 66/73 $(90.4 \%)$ as defined above (see Methods). Three patients had atrophic kidneys that failed to recover function after surgery. One patient had recurrent pyelonephritis and subsequently underwent a simple nephrectomy 12 months later. One patient had resolution of pain but no improvement of hydronephrosis on $\mathrm{U} / \mathrm{S}$, and 2 patients continue to have pain. These patients are currently being treated conservatively and have stable renal function.

Pyelolithotomy was performed on 6 patients concomitantly with the LP procedure, without difficulty. In one case, several stones were deep within the calyces and were removed endoscopically at a later date. For these cases, the average operative time was extended by 36 minutes.

\section{Discussion}

With success rates that are consistently over $90 \%$, Anderson-Hynes open pyeloplasty remains the gold standard for UPJO. ${ }^{1}$ Over the last 20 years, however, great strides have been made in minimally invasive treatment options for UPJO, and various procedures now exist. Minimally invasive treatments now include percutaneous antegrade and retrograde endopyelotomy as well as various LP techniques. The advantages of minimally invasive procedures over traditional open pyeloplasties include a decreased hospital stay and a significant reduction in morbidity that includes a large incision, associated pain and risk of nerve injury. Investigators at Vanderbilt University recently implemented a clinical pathway to reduce length of stay in patients undergoing LP and were able to discharge patients on postoperative day 1 while maintaining a high level of satisfaction. ${ }^{14}$ The possibilities of less patient morbidity, reduced length of stay and lower hospital costs make minimally invasive techniques an attractive option for physicians and patients alike.

Comparison of antegrade and retrograde endopyelotomy showed no significant difference in success rates between the 2 procedures, although retrograde endopyelotomy is associated with shorter hospital stays, fewer complications and lower costs. ${ }^{15}$ The 2 most common retrograde approaches are hot-wire balloon endopyelotomy and holmium laser endopyelotomy. Success rates are comparable between the 2 retrograde techniques, although holmium laser endopyelotomy is associated with fewer hemorrhagic complications in one series. ${ }^{16}$ Initial followup of patients undergoing endopyelotomy yielded success rates close to those of open pyeloplasty, ${ }^{17}$ but a recent long-term review at a single institution showed respective success rates of $63 \%$, $55 \%$ and $41 \%$ for $3-$, 5- and $10-y e a r$ recurrencefree survival. Comparatively, the same institution's 3-, 5- and 10-year recurrence-free survival for pyeloplasty (open and laparoscopic combined), were $85 \%, 80 \%$ and $75 \%$, respectively. ${ }^{2}$ Another study showed a significant reduction in success rates in patients with significant hydronephrosis and crossing vessels, limiting its effectiveness to select patients only..$^{18}$ These studies raise questions about the durability of endopyelotomy in the long term.

LP offers success rates that are comparable to open pyeloplasty without the added morbidity and length of stay. Excellent outcomes have been reported for

\begin{tabular}{lc}
\hline Table 3. Patient outcomes & \\
\hline Outcome & $\begin{array}{c}\text { No. (and \%) of } \\
\text { patients }\end{array}$ \\
\hline $\begin{array}{l}\text { Successful } \\
\text { Improved pain and imaging }\end{array}$ \\
$\begin{array}{l}\text { Unsuccessful } \\
\text { No improvement in renal function }\end{array}$ \\
$\begin{array}{l}\text { Pain } \\
\text { Infection }\end{array}$ \\
No improvement on intravenous pyelogram & $3 / 73(4.1)$ \\
\hline
\end{tabular}


both transperitoneal and retroperitoneal LP. In their retroperitoneal series of LPs, Moon and colleagues ${ }^{5}$ listed the advantages of the retroperitoneal approach, which include less dissection to reveal the UPJ, avoiding problems associated with exposing the peritoneal cavity and easier identification of crossing vessels. ${ }^{5}$ The transperitoneal approach offers a larger work space, which makes dissection, anastomosis and ureteral displacement easier, particularly if a crossing vessel is encountered. ${ }^{4}$ Because we have achieved excellent results with the transperitoneal dismembered approach, we will continue using this technique at our institution.

Compared with retrograde stent insertion before surgery, the antegrade approach is quicker to do intraoperatively. We have also found dissection of the renal pelvis and visualization of the site of the obstruction easier with the antegrade approach because the renal pelvis remains distended; additionally, the posterior anastomosis is easier to accomplish because the stent is placed after its completion. Stent removal was initially undertaken 6 weeks after surgery, but because of stent morbidity, one of the surgeons changed this removal to 2 weeks after surgery. Three of the surgeons still remove the stent between 4 and 6 weeks after surgery. There has not been any difference in outcome between the 2 removal times.

Our overall success rate of $90.4 \%$ is consistent with that achieved with open pyeloplasty. Patients with partial pain resolution are being followed conservatively and undergo serial imaging to document further decline or obstruction. In this retrospective study, we evaluated symptoms subjectively, which limits accuracy, and for future evaluations, a validated pain scale may be used.

Urolithiasis and multiple stones are common conditions associated with UPJO. For the management of UPJO, the LP has better long-term success rates than endopyelotomy. ${ }^{2,18}$ Thus in the case of a large renal pelvis and stone, our first choice is LP with concomitant pyelolithotomy in preference to percutaneous antegrade endopyelotomy with percutaneous nephrolithotripsy. Pyelolithotomy at the time of LP did not result in any additional complications or morbidities for those patients who required such a procedure, but operating times increased by an average of 36 minutes.

The initial degree of hydronephrosis and any improvement on subsequent imaging was taken from the interpretation in the radiologist's report.
This was a limitation of our retrospective study because not all characteristics of the obstruction were addressed consistently. Better imaging details and consistency can be obtained if the radiologists involved are blinded to the images and given specific criteria to comment on for all imaging before and after surgery. Computed tomography (CT) scans were done for some patients, but the results are not included because not all surgeons performed CT scans on their patients to look for the source of obstruction. Although a CT scan and knowing that crossing vessels are present is useful for the decision to undergo endopyelotomy, our patients were to undergo LP. During LP, one looks for a crossing vessel during dissection; hence, CT confirmation is not necessary.

Past studies have shown that prolonged obstruction can lead to irreversible renal damage as determined by an assessment of renal function with a renal scan. ${ }^{19,20}$ Indeed, 3 of our patients had no improvement in their renal function after surgery. Two of these 3 patients continue to have mild flank discomfort. With the additional risks of hypertension and infection associated with an atrophic kidney, these patients might have benefited more from laparoscopic nephrectomy than from LP. To help surgeons decide between the 2 procedures, a preoperative renal function cut-off needs to be established. One study looking at renal function before and after pyeloplasty showed no improvement in patients with a preoperative renal function of less than $20 \%$ (9 patients), ${ }^{19}$ and in another study, only 2 of 10 patients with preoperative renal function of less than $30 \%$ improved postoperatively. ${ }^{20}$ Our 3 patients all had renal functions of less than $25 \%$ on preoperative renal scan, but another patient with a preoperative renal function of $26 \%$ subsequently improved to $36 \%$ postoperatively. We believe that the recovery of renal function is unlikely for patients with a preoperative renal function of less than $25 \%$, and nephrectomy could be a possible option for these patients.

The success of LP has led to many innovations to improve operating time and reduce difficulty. Araki and colleagues ${ }^{8}$ reported using an EndoGIA stapler (United States Surgical Corp.) to remove the redundant renal pelvis and reduce operating time. The primary concern with using an EndoGIA stapler is the potential for urolithiasis with the staples acting as the nidus. Thus far, this has not occurred in the 6 patients that Araki and colleagues 
Dong et al.

have followed for 5 years. ${ }^{8}$ Another novel approach is the use of robotic assistance to aid with intracorporeal suturing. This had initially shown an excellent reduction in mean operative times. ${ }^{9}$ However, more recent data have shown no improvement in suturing speed or quality for a surgeon who is already skilled in intracorporeal suturing, although it does add substantial cost. ${ }^{10}$ Other techniques for approximating the anastomosis, such as the use of fibrin glue or laser welding, have not fared well in porcine models and lack longterm follow-up studies. ${ }^{11}$

Endourological treatment for UPJO is less technically demanding and requires less time to perform than LP. New methods have also been developed to improve the success rates of endopyelotomy while preserving its advantages over LP. The group at the Cleveland Clinic recently described their percutaneous endopyeloplasty procedure, which consists of a Fenger-plasty done percutaneously. ${ }^{21}$ The initial results of this procedure are promising, but this study was limited by the number of patients as well as by followup time. ${ }^{22}$ The group has further extended their endourological technique by performing a dismembered pyeloplasty percutaneously and coining the term percutaneous intrarenal reconstructive surgery (PIRS) to describe endourological surgery consisting of complex dissection and reconstruction of intrarenal and retroperitoneal structures. ${ }^{23}$ These new techniques offer promising treatment options for UPJO but will need to be validated in larger and long-term studies.

\section{Conclusion}

LP has consistently achieved success rates comparable to those of open pyeloplasty. The reduced patient morbidity and length of stay offer considerable advantages over open procedures, and LP should be first-line treatment for UPJO undertaken by experienced laparoscopic surgeons. Our institution has a success rate comparable tothat of other centres that have published results, and no revisions have been done to date.

*Medical Student, †Associate Professor, †Assistant Professor, McMaster University, St. Joseph's Hospital, Hamilton, Ont., §Fellow and IResident, Division of Urology, St. Joseph's Hospital, Hamilton, Ont.

This article has been peer reviewed.

Competing interests: None declared.

\section{References}

1. O'Reilly PH, Brooman PJ, Mak S, et al. The long-term results of Anderson-Hynes pyeloplasty. BJU Int 2001;87:287-9.

2. Dimarco DS, Gettman MT, McGee SM, et al. First prize: long-term success of antegrade endopyelotomy compared with pyeloplasty at a single institution. J Endourol 2006;10: 707-12.

3. Schussler WW, Grune MT, Tecuanhuey LV, et al. Laparoscopic dismembered pyeloplasty. J Urol 1993;150:1795.

4. Inagaki T, Kavoussi LR, Jarrett TW, et al. Laparoscopic pyeloplasty: current status. BJU Int 2005;95(suppl 2):102-5.

5. Moon DA, E-Shazly MA, Chang CM, et al. Laparoscopic pyeloplasty: evolution of a new gold standard. Urology 2006;67:932-6.

6. Davenport K, Minervini A, Timoney AG, et al. Our experience with retroperitoneal and transperitoneal laparoscopic pyeloplasty for pelvi-ureteric junction obstruction. Eur Urol 2005; 48:973-7.

7. Manikandan R, Saad A, Bhatt Rl, et al. Minimally invasive surgery for pelviureteral junction obstruction in adults: a critical review of the options. Urology 2005;65:422-32.

8. Araki H, Ono Y, Hattori R, et al. Laparoscopic pyeloplasty using endoscopic GIA stapler for ureteropelvic junction obstruction. J Endourol 2005; 19:143-6.

9. Peschel R, Neururer R, Bartsch G, et al. Robotic pyeloplasty: technique and results. Urol Clin North Am 2004:31:737-41.

10. Link RE, Bhayani SB, Kavoussi LR. A prospective comparison of robotic and laparoscopic pyeloplasty. Ann Surg 2006;243:486-91.

11. Barrieras D, Reddy PP, McLorie GA, et al. Lessons learned from laser tissue soldering and fibrin glue pyeloplasty in an in vivo porcine model. J Urol 2000;164:1106-10.

12. Hussain A, Whelan P, Piercey K, et al. McMaster experience with laparoscopic pyeloplasty. Can J Urol 2004;3:2299-302.

13. Obeid AA, Whelan JP, Piercey K, et al. Laparoscopic pyeloplasty with intra-operative antegrade placement of the double J stent [abstract]. $57^{\text {th }}$ Annual Meeting of the Canadian Urological Association; 2001 Jun 24-27; Toronto, Ont.

14. Webster TM, Baumgartner R, Herrell SD, et al. A clinical pathway for laparoscopic pyeloplasty decreases length of stay. J Urol 2005;173:2081-4.

15. Shalhav AL, Giusti G, Elbahnasy AM, et al. Adult endopyelotomy: impact of etiology and antegrade versus retrograde approach on outcome. J Urol 1998;160:685-9.

16. Ponsky LE, Streem SB. Retrograde endopyelotomy: a comparative study of hot-wire balloon and ureteroscopic laser. J Endourol 2006;20:823-6.

17. Kletscher BA, Segura J, LeRoy A, et al. Percutaneous antegrade endoscopic pyelotomy: review of 50 consecutive cases. J Urol 1995;153:701-3.

18. Van Cangh PJ, Wilmart JF, Opsomer RJ, et al. Long-term results and late recurrence after endoureteropyelotomy: A critical analysis of prognostic factors. J Urol 1994; 151:934-7.

19. Tripathi $M$, Kumar $R$, Chandrashekar $N$, et al. Diuretic radionuclide renography in assessing Anderson-Hynes pyeloplasty in unilateral pelviureteric junction obstruction. Hell J Nucl Med 2005;8:154-7

20. Ortapamuk H, Naldoken S, Tekdogan UY, et al. Differential renal function in the prediction of recovery in adult obstructed kidneys after pyeloplasty. Ann Nucl Med 2003;17:663-8

21. Desai MM, Gill IS, Carvalhal EF, et al. Percutaneous endopyeloplasty: a novel technique. J Endourol 2002;16:431-43.

22. Desai MM, Desai MR, Gill IS. Endopyeloplasty versus endopyelotomy versus laparoscopic pyeloplasty for primary ureteropelvic junction obstiuction. Urology 2004;64:16-21

23. Sharp DS, Desai MM, Molina WR, et al. Dismembered percutaneous endopyeloplasty: a new procedure. J Endourol 2005;19:210-7.

Correspondence: Dr. Edward D. Matsumoto, Assistant Professor, Division of Urology, McMaster University, St. Joseph's Hospital. 50 Charlton Ave. E., G343, Hamilton ON L8N 4A6; matsumo@mcmaster.ca 